\title{
Entangled photon assisted multidimensional nonlinear optics of exciton-polaritons
}

Cite as: J. Appl. Phys. 128, 113102 (2020); https://doi.org/10.1063/5.0012754

Submitted: 04 May 2020. Accepted: 03 September 2020. Published Online: 18 September 2020

Arunangshu Debnath (iD), and Angel Rubio (iD

\section{COLLECTIONS}

Paper published as part of the special topic on Materials for Quantum Technologies: Computing, Information, and Sensing
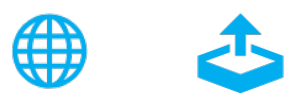

\section{ARTICLES YOU MAY BE INTERESTED IN}

Probing exciton/exciton interactions with entangled photons: Theory

The Journal of Chemical Physics 152, 071101 (2020); https://doi.org/10.1063/1.5139197

Cleavable magnetic materials from van der Waals layered transition metal halides and chalcogenides

Journal of Applied Physics 128, 110901 (2020); https://doi.org/10.1063/5.0023729

Theoretical modeling of triboelectric nanogenerators (TENGs)

Journal of Applied Physics 128, 111101 (2020); https://doi.org/10.1063/5.0020961

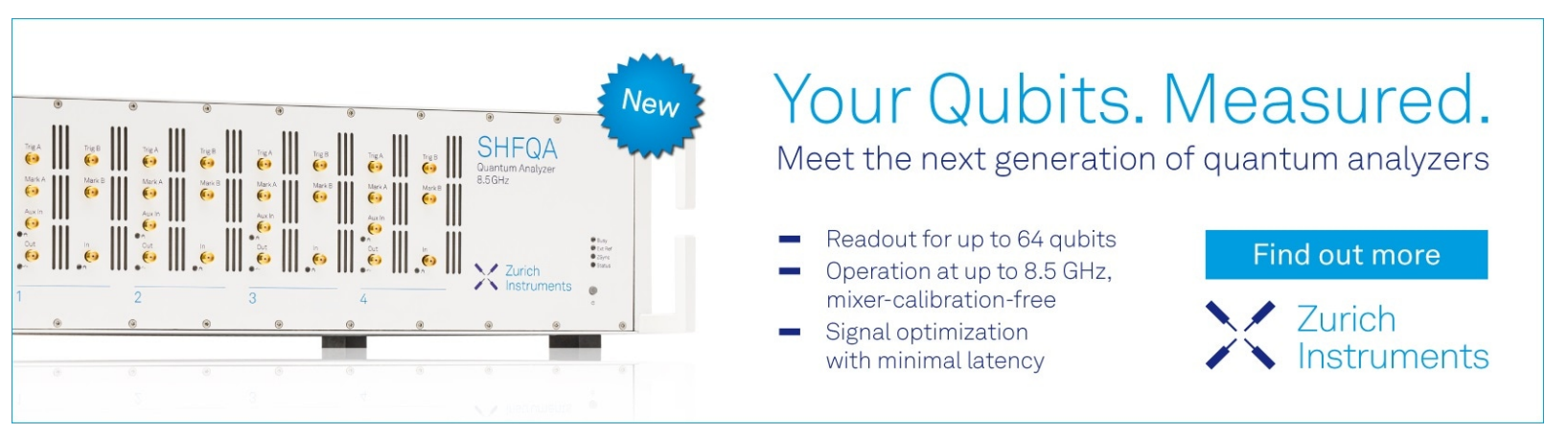




\title{
Entangled photon assisted multidimensional nonlinear optics of exciton-polaritons
}

Cite as: J. Appl. Phys. 128, 113102 (2020); doi: 10.1063/5.0012754

Submitted: 4 May 2020 - Accepted: 3 September 2020 .

Published Online: 18 September 2020

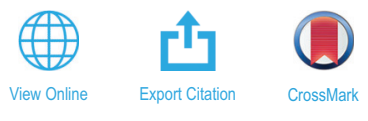

Arunangshu Debnath $^{1, a)}$ (iD and Angel Rubio ${ }^{1,2, b)}$ (iD

\section{AFFILIATIONS}

${ }^{1}$ Max Planck Institute for the Structure and Dynamics of Matter and Center for Free-Electron Laser Science, Luruper Chaussee 149, 22761 Hamburg, Germany

${ }^{2}$ Center for Computational Quantum Physics (CCQ), The Flatiron Institute, 162 Fifth Avenue, New York, New York 10010 , USA

Note: This paper is part of the special collection on Materials for Quantum Technologies: Computing, Information, and Sensing.

a) Author to whom correspondence should be addressed: arunangshu.debnath@desy.de

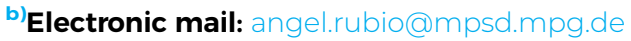

\begin{abstract}
We present a theoretical formulation of the frequency domain multidimensional pump-probe analog spectroscopy, which utilizes the spectral-temporal entanglement features of the biphoton sources. It has been shown, via a compact multi-time, convolutional Green's function expression and the accompanying numerical simulations, that utilizing the correlation properties of non-classical sources offers a viable scheme for the exploration of dissipative kinetics of the cavity confined quantum aggregates. The cooperative and competitive modifications brought in by the photonic cavity mode and the auxiliary vibrational modes into the scattering and dephasing properties of the excitonpolaritons have been explored via their signatures in the multidimensional correlation maps. The study offers a new parameter window for the investigation of the dynamical polariton characteristics and warrants the usage of multi-mode entanglement properties of the external photonic sources in future studies.
\end{abstract}

Published under license by AIP Publishing. https://doi.org/10.1063/5.0012754

\section{INTRODUCTION}

Over the past few decades, the ability to engineer and control multi-mode entanglement of spatial-temporal modes of the photonic sources gave rise to a plethora of applications in quantum communications, metrology, computation, and precision measurements. ${ }^{1-5}$ Such studies can also be extended to the domain of the characterization of unknown matter properties, where the non-classical correlation features of the optical modes induce controlled dynamical evolution of the matter modes, thereby emphasizing their scope as spectroscopic probes. ${ }^{6-10}$ In such studies, the resultant spectral features of the matter modes crucially depend on the windowed dynamics in the joint time-frequency space, which were controllably induced. Depending on their generation processes, the photonic modes can be entangled in temporal-spectral, polarization, or the orbital angular momentum degrees of freedom. ${ }^{1-13}$ For the pump-probe analog proposed in this communication, we focus on the biphoton sources having non-classical spectral-temporal properties in the optical regime.
Traditionally, the optical pump-probe techniques or their multi-pulse generalizations have attempted to decipher the quasiparticle correlation dynamics by carrying out experiments either by pumping the matter modes via temporally strobed laser pulses or by pumping via coincident multi-frequency laser pulses. These techniques, which probe the matter dynamical responses, are collectively referred to as multidimensional coherent spectroscopies (MDCSs). ${ }^{14,15}$ The origin of the dynamical response of the matter modes is related to the contributions originating from the timedependent matter polarization, which contains relevant information about the interactions among the participating matter modes. The signal thus generated has the temporal and spectral features related to the mode correlation and dephasing encoded into it. ${ }^{16-18}$ The classical field-assisted (e.g., shaped laser pulse assisted) MDCS techniques provide an accessible control over the spectroscopic parameter space via the pulse bandwidths, central frequencies, relative phases, and inter-pulse delays. The inter-pulse intervals offer, via multidimensional Fourier transforms, a convenient visualization window for the correlations among matter modes in the frequency 
space. In contrast, the entangled sources allow enhanced control of the parameters enabling direct access to the biphoton wavefunctions. The fact that the corresponding biphoton amplitude properties can be manipulated brings several advantages to their plausible use in exploring the mode correlations in the multidimensional frequency space. Moreover, the quantum field-induced signals scale favorably with the field intensities, making them suitable for studying the transient cavity mediated phenomenology in the low-energy regime. In this communication, we focus on the transmitted mode pump-probe analog while treating their respective frequencies as the parametric scanning variables for the generation of the spectra. $^{19-23}$

In a distinct but concurrent development within the field of the cavity, quantum electrodynamics have shown the ability of the spatially confined photonic modes to interact with the localized matter transitions and modify the dynamical energy landscape. Collective multi-exciton processes, e.g., exciton-migrations, longrange cavity mediated exciton transfer, proximity induced excitonexciton scattering occurring in the interacting exciton harvesting chromophores, namely, quantum aggregates, offer several dynamical features that could change drastically in the presence of the cavity mode. The ability to engineer optical cavity modes that interact with the collective excitons and alter their delocalization properties offer an additional investigative tool for the cooperative exciton dynamics in these systems. Toward this aim, the cavity mediated control of the coherent excitonic transport properties in the quantum aggregates remains a coveted goal. ${ }^{24-32}$ This communication aims to leverage the static tunability of the cavity to generate the exciton-polariton sideband modes and investigate the emergent mode correlation dynamics in the presence of phonon induced dissipation using the entangled biphoton sources (Fig. 1).
In Sec. II, we propose the cavity-exciton-phonon Hamiltonian that serves as a prototypical spectroscopic model for this study and introduces the polariton basis and the relevant polariton Green's functions. In Sec. III, we present a brief description of the entangled photon generation protocols followed by the evaluation of the proposed entangled biphoton enhanced pump-probe analog signal and the presentation of the numerical simulations. Section IV contains a brief discussion of the presented work along with an outlook that summarizes the article.

\section{DISSIPATIVE EXCITON-POLARITON KINETICS}

\section{A. Hamiltonian}

The quantum aggregates typically consist of few electronic sub-units, i.e., sites which, under the optical excitations, display coherent and incoherent exciton transfer across the sites. The exciton kinetics in these systems, under low-intensity optical excitations, can be well-described via the Frenkel exciton Hamiltonian and the associated generalized master equation. ${ }^{33}$ The nature of exciton energy transfer in the quantum aggregates is principally governed via the mutual interplay between the coulomb correlations and vibrational dephasing. The former gives rise to exciton delocalization while the latter is responsible for the collective dephasing of excitons. In the presence of the cavity interaction, the exciton transfer pathways across the aggregates are significantly altered. Often, it gives rise to counter-intuitive observations such as dissipation-less energy migration or extraordinary spatial range exciton transfer phenomenology. ${ }^{34-36}$ In this work, we focus on a functional prototype representing the light-harvesting complex, LHC-II, which has been under investigation for its efficient exciton transfer under ambient conditions. ${ }^{37-40}$
(A)

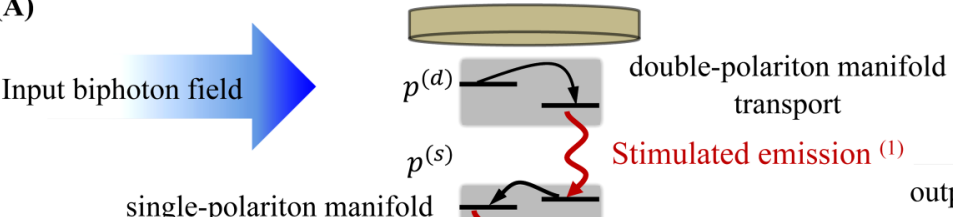
transport
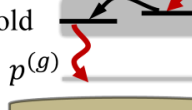

Stimulated emission (2) output signal field

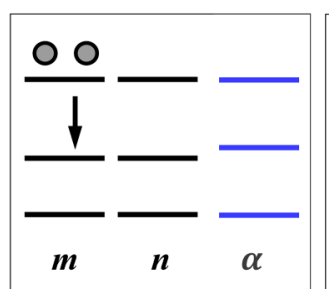

(B1)

double-exciton deexcitation

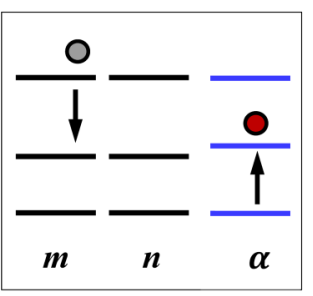

(B2)

cavity mode excitation; doubleexciton stimulated emission

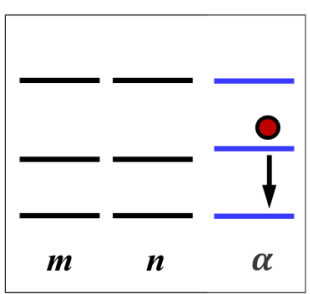

(B3)

cavity mode deexcitation

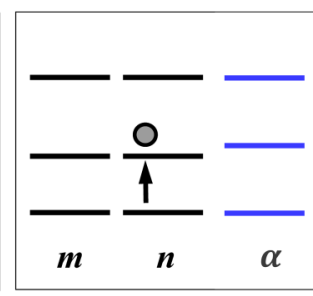

(B4)

exciton formation
FIG. 1. (a) Scheme for a prototypical process that is expected to be revealed by the proposed signal measurement technique in direct matter pumping configuration in the presence of cavity. It involves the phonon-assisted transport between the two states in the double polariton manifold, followed by the inter-manifold stimulated emission and transport between states in the single polariton manifold. It undergoes further stimulated emission, which contributes to the observed signal. The interpretive nature of the processes in the site basis $\left|N_{m}, N_{n}, N_{\alpha}\right\rangle$ of two excitonic sites $(m, n)$ and one cavity mode $(\alpha)$ (with excitation numbers $N_{i}$ ) is illustrated in (b1)-(b4). The cavity mediated pathways proceed via $|2,0,0\rangle$, $|1,0,1\rangle,|0,0,1\rangle$, and $|0,1,0\rangle$, resulting in the conversion of a local double exciton into a single exciton accompanied by their complementary cavity and external radiation mode processes. 
In order to construct the combined exciton-cavity-phonon Hamiltonian, we systematically introduce the individual Hamiltonian components and describe the approximations made in the following. Throughout this work, the $\hbar$ is set to unity. The exciton site Hamiltonian $H_{s}$ is given by $H_{s}=\sum_{m, n=1}^{N_{s}}$ $\left(E_{m} \delta_{m n}+J_{m n}\right) B_{m}^{\dagger} B_{n}+\sum_{m, n, k, l=1}^{N_{s}} K_{m n k l} B_{m}^{\dagger} B_{n}^{\dagger} B_{k} B_{l}$, where $B_{m}\left(B_{m}^{\dagger}\right)$ are the exciton creation (annihilation) operators (at the $m$ th site), which follows the deformed boson statistics $\left[B_{m}, B_{n}\right]=$ $\left[B_{m}^{\dagger}, B_{n}^{\dagger}\right]=0$ and $\left[B_{m}, B_{n}^{\dagger}\right]=\delta_{m n}\left(1-q_{m}^{(1)} B_{m}^{\dagger} B_{m}^{\dagger} B_{m} B_{m}\right)$, where $q_{m}=3 / 2$ has been set for this work. ${ }^{41,42}$ The exciton operator statistics governs the nature of the different nonlinear excitonic processes that can be described within this formalism. Within its monomeric form, LHC-II is composed of $N_{s}=14$ individual chromophores (consisting of chlorophyll molecules) that act as a host for the single and double excitons. The on-site excitation energies are given by $E_{m}$, and the inter-site Coulomb mediated hopping, given by $J_{m n}$, account for the exciton migration. The corresponding values are obtained from the semi-empirical simulation performed in Refs. 43 and 44, which had demonstrated simultaneous spectral fit with the experimentally obtained linear absorption, fluorescence, and the circular dichroism spectra. The multi-exciton interaction term $\left(K_{m n k l}\right)$ describes plausible higher-order excitonic processes and accounts for the energy shifts for the local multi-exciton state energies. In the present work, we approximate this term, limiting ourselves to contributions involving $K_{m n m n}\left(K_{m n n m}\right)$ and $K_{m m m m}$, which accounts for the non-local energy shifts, $\Delta_{m n}^{(1)}=4 K_{m n m n}=4 K_{m n n m}$ and local energy shifts, $\Delta_{m m}^{(2)}=2 K_{m m m m}$ to the double exciton states, respectively. The corresponding numerical values are sampled from two separate Gaussian distributions with the width $\sigma\left(\mathrm{cm}^{-1}\right)=50.0$ using stochastic sampling. The approximated exciton Hamiltonian can be expressed as $\tilde{H}_{s}=\sum_{m, n=1}^{N_{s}}\left(E_{m} \delta_{m n}+J_{m n}\right) \quad B_{m}^{\dagger} B_{n}+$ $\sum_{m=1}^{N_{s}}\left(\Delta_{m m}^{(2)} / 2\right) B_{m}^{\dagger} B_{m}^{\dagger} B_{m} B_{m}+\sum_{m, n=1 ; m \neq n}^{N_{s}}\left(\Delta_{m n}^{(1)} / 2\right) B_{m}^{\dagger} B_{n}^{\dagger} B_{m} B_{n}$.

The exciton-phonon interactions originate from the interand intra-molecular vibrational motions associated with the nuclear degrees of freedom of the aggregate. The corresponding normal modes of the low-energy displacement degrees of freedom of the collective vibrational coordinates, i.e., the phonon modes, are responsible for exciton dephasing and relaxation. The phonons, represented by the free phonon Hamiltonian $H_{b}$, is formally represented by an infinite set of Harmonic oscillators. The excitonphonon interactions, taken in the site-uncorrelated, local form are characterized by the distribution of the corresponding coupling functions, $\bar{g}_{k}$. The combined phonon and exciton-phonon interaction Hamiltonian can be presented as $H_{b}+H_{s b}=\sum_{k} v_{k}\left(b_{k}^{\dagger} b_{k}+\right.$ $1 / 2)+\sum_{m, k} \bar{g}_{k}\left(b_{k}+b_{k}^{\dagger}\right) B_{m}^{\dagger} B_{m}$ where $v_{k}$ is the mode frequency associated with the $k$ th normal mode whose creation (annihilation) operators are denoted via $b_{k}^{\dagger}\left(b_{k}\right)$. The phonon operators follow the free-boson commutation relations, $\left[b_{k}, b_{k^{\prime}}\right]=\left[b_{k}^{\dagger}, b_{k^{\prime}}^{\dagger}\right]=0$ and $\left[b_{k}, b_{k^{\prime}}^{\dagger}\right]=\delta_{k k^{\prime}}$. The magnitude of the coupling strengths of the local single excitons are taken as identical while that of the double excitons are taken as twice as large of the former. Consequently, within this model, the single and the double excitons are coupled to a common set of phonon modes which can be characterized by the site-independent spectral function, $J(\omega)=\pi \sum_{k}\left|\bar{g}_{k}\right|^{2}\left(\delta\left(\omega-v_{k}\right)\right.$ $\left.-\delta\left(\omega+v_{k}\right)\right)$ from which the spectral density function is obtained in the continuum frequency limit. The spectral density function, presented as $J(\omega)=2 \lambda_{0}\left(\gamma_{0} \omega\right) /\left(\omega^{2}+\gamma_{0}^{2}\right)+\sum_{j=1}^{N_{b}} 2 \lambda_{j}\left(v_{j}^{2} \gamma_{j} \omega\right) /$ $\left(\left(v_{j}^{2}-\omega^{2}\right)^{2}+\omega^{2} \gamma_{j}^{2}\right)$ consisted of $N_{b}=48$ multi-mode Brownian oscillators and one over-damped oscillator. The number of multimode Brownian oscillators $\left(N_{b}\right)$, the respective spectral shift parameters $\left(\lambda_{0}, \lambda_{j}=v_{j} \Upsilon_{j}\right.$, where $v_{j}$ and $\Upsilon_{j}$ are the $j$ th oscillator frequencies and Huang-Rhys parameters, respectively), and the damping parameter $\gamma_{0}, \gamma_{j}$ that are required to optimally describe the equilibrium spectral density function have been obtained via a systematic spectral fitting procedure in the work of Novoderezhkin et al. ${ }^{32,43}$ The numerical values corresponding to those parameters are enlisted in Appendix B.

The combined cavity and the cavity-exciton interaction Hamiltonian can be given by $H_{c}+H_{s c}=\sum_{\alpha} \omega_{\alpha}\left(a_{\alpha}^{\dagger} a_{\alpha}+1 / 2\right)$ $+\sum_{m, \alpha} g_{c ; m, \alpha}^{(1)}\left(a_{\alpha} B_{m}^{\dagger}+a_{\alpha}^{\dagger} B_{m}\right)+g_{c ; \alpha}^{(2)}\left(a_{\alpha}+a_{\alpha}^{\dagger}\right)^{2}$, where we use the $g_{c ; m, \alpha}^{(1)}$ and $g_{c ; \alpha}^{(2)}$ to denote the cavity-exciton coupling strengths originating from resonant dipolar transitions and the non-resonant energy renormalization induced shifts, respectively. The cavity photonic modes are described in the oscillator basis with the corresponding creation (annihilation) operators denoted as $a_{\alpha}^{\dagger}\left(a_{\alpha}\right)$, for the $\alpha$ th mode. Within the scope of this study, a single mode limit, i.e., $\alpha=1$, is taken, which yields the single cavity mode with the fundamental frequency denoted by $\omega_{c}$ and uniform coupling strengths $g_{c ; m, \alpha}^{(1)}=g_{c}$. The cavity, notably, accommodates two-photon excitations, which are capable of resonantly interacting with multiexciton processes. The cavity-exciton interaction strength is determined by the mode volume of the cavity and the quality factor. ${ }^{45-47}$ The effect arising from the $g_{c ; \alpha}^{(2)}$ could be significant where the exciton-cavity coupling strengths become comparable to the bare Rabi frequencies of the multi-exciton system reaching the ultra-strong regime. ${ }^{46,48,49}$ Considering the parameters regime treated in this communication, i.e., the range of weak to moderate exciton-cavity coupling strengths, this term has been neglected in the subsequent analysis. The limiting form of the single mode cavity and the cavityexciton interaction Hamiltonian is given by $\tilde{H}_{c}+\tilde{H}_{s c}=$ $\omega_{c}\left(a^{\dagger} a+1 / 2\right)+\sum_{m} g_{c}\left(a B_{m}^{\dagger}+a^{\dagger} B_{m}\right)$. Combining the individual components together, i.e., $H=\tilde{H}_{s}+H_{b}+H_{s b}+\tilde{H}_{c}+\tilde{H}_{s c}$, we obtain the working Hamiltonian as

$$
\begin{aligned}
H= & \sum_{m, n=1}^{N_{s}}\left(E_{m} \delta_{m n}+J_{m n}\right) B_{m}^{\dagger} B_{n}+\sum_{m=1}^{N_{s}}\left(\Delta_{m m}^{(2)} / 2\right) B_{m}^{\dagger} B_{m}^{\dagger} B_{m} B_{m} \\
& +\sum_{m, n=1 ; m \neq n}^{N_{s}}\left(\Delta_{m n}^{(1)} / 2\right) B_{m}^{\dagger} B_{n}^{\dagger} B_{m} B_{n} \\
& +\sum_{k} \hbar v_{k}\left(b_{k}^{\dagger} b_{k}+1 / 2\right)+\sum_{m, k} \bar{g}_{k}\left(b_{k}+b_{k}^{\dagger}\right) B_{m}^{\dagger} B_{m} \\
& +\omega_{c}\left(a^{\dagger} a+1 / 2\right)+\sum_{m} g_{c}\left(a B_{m}^{\dagger}+a^{\dagger} B_{m}\right) .
\end{aligned}
$$

The cavity modulated exciton kinetics can be explored by introducing the polariton basis, which is obtained by exact diagonalization of the 
field-free Hamiltonian subspace, $\tilde{H}_{p}=\tilde{H}_{s}+\tilde{H}_{c}+\tilde{H}_{s c}$ to obtain $U_{p}^{-1} \tilde{H}_{p} U_{p}=H_{p}=\sum_{n=g, s, d} E_{p^{(n)}} X_{p^{(n)} p^{(n)}}$, where the operators $X_{p^{(n)} p^{(n)}}=\left|p^{(n)}\right\rangle\left\langle p^{(n)}\right|$ are the projectors onto the diagonal elements of the polariton Hamiltonian $H_{p}$ corresponding to excitation conserving polariton manifolds denoted by index $n$. The three energetically distinct sectors, namely, ground, single, and double polariton manifolds corresponding to $n=g, s, d$ contains $N_{p^{(g)}}=1, N_{p^{(s)}}=15$, and $N_{p^{(d)}}=120$ polariton states, respectively. The proposed spectroscopic technique utilizing entangled biphotons, in the parameter regime of interest, interact only via two-quantum interactions, which allows the joint-excitation manifold to be truncated at the level of the double polaritons. The spectral weights of the resultant delocalized polariton states have respective contributions from both the exciton and cavity modes, as determined by the exciton-cavity coupling matrix elements. For the simulation, we vary the cavity mode frequency and the coupling strength parameters according to $\omega_{c}^{\mathrm{I}}\left(\mathrm{cm}^{-1}\right)=15.0 \times 10^{3}, \quad \omega_{c}^{\mathrm{II}}\left(\mathrm{cm}^{-1}\right)=15.4 \times 10^{3}, \quad \omega_{c}^{\mathrm{III}}\left(\mathrm{cm}^{-1}\right)=$ $15.7 \times 10^{3}$ and $g_{c}^{\mathrm{I}}\left(\mathrm{cm}^{-1}\right)=0.1 \times 10^{3}, g_{c}^{\mathrm{II}}\left(\mathrm{cm}^{-1}\right)=0.25 \times 10^{3}$, $g_{c}^{\text {III }}\left(\mathrm{cm}^{-1}\right)=0.5 \times 10^{3}$, which generates nine distinct cases (hereafter denoted as, e.g., $\left\{\omega_{c}^{(i)}, g_{c}^{(j)}\right\}$ with $i, j$ running over I-III) for the pairwise combination of the parameters. In Fig. 2, we present the single polariton energy levels while the central frequencies are scanned for three parametric cases of the exciton-cavity coupling strengths, i.e., $g_{c}^{\mathrm{I}-\mathrm{III}}$. The emergent sidebands, along the three vertical lines, at non-equidistant energies from the central spectral region are the states whose dynamics are of central interest to the present study. With the increase of exciton-cavity coupling strengths, the sidebands appear at distant energies. These two observations, combined together, affirm that the exciton-cavity hybridization dominantly takes around the resonances, as expected.

The external biphoton-polariton interaction is treated within the dipole approximation and the corresponding Hamiltonian $H_{\text {int }}(t)=E(t) \tilde{X}^{\dagger}(t)+E^{\dagger}(t) \tilde{X}(t)$, in the interaction representation, is defined as

$$
\begin{aligned}
H_{\text {int }}(t)= & \sum_{j} \sum_{n, n^{\prime}=g, s, d ; n \neq n^{\prime}}\left(\sqrt{2 \pi \omega_{j} / V}\right) a_{j} e^{-i \omega_{j} t} d_{p^{(n)} p^{\left(n^{\prime}\right)}} X_{p^{(n)} p^{\left(n^{\prime}\right)}} \\
& \times \exp \left(i \omega_{p^{\left(n^{\prime}\right)} p^{(n)}} t\right)+\text { h.c. }
\end{aligned}
$$

In the above, we have defined the dipole weighted inter-manifold polariton transition operators, $\tilde{X}=\sum_{n, n^{\prime}=g, s, d ; n \neq n^{\prime}} d_{p^{(n)} p^{\left(n^{\prime}\right)}} X_{p^{(n)} p^{\left(n^{\prime}\right)}}=$ $d_{p^{(n)} p^{\left(n^{\prime}\right)}}\left|p^{(n)}\right\rangle\left\langle p^{\left(n^{\prime}\right)}\right|$ and introduced the decomposition of the entangled biphoton field $E(t)=\sum_{j} \sqrt{\left(2 \pi \omega_{j} / V\right)} a_{j} \exp \left(-i \omega_{j} t\right)+$ h.c. in the external photon modes $a_{j}$ has been introduced. The mode quantization volume and the Fourier expansion frequency for the photon modes are denoted as $V$ and $\omega_{j}$, respectively.

\section{ENTANGLED BIPHOTON ASSISTED MULTIDIMENSIONAL SPECTROSCOPY}

In the prescribed measurement configuration, i.e., in Fig. 3, the signal is detected in the transmitted mode configuration and dispersed in two parametric frequency dimensions $\left(\Omega_{2}, \Omega_{1}\right)$ after one of the two beams pass through the cavity, which hosts the quantum aggregate. The pump-probe analog signal is defined by taking all possible polariton-biphoton interactions into account where the role of pump and probe originating from either of the two beams are

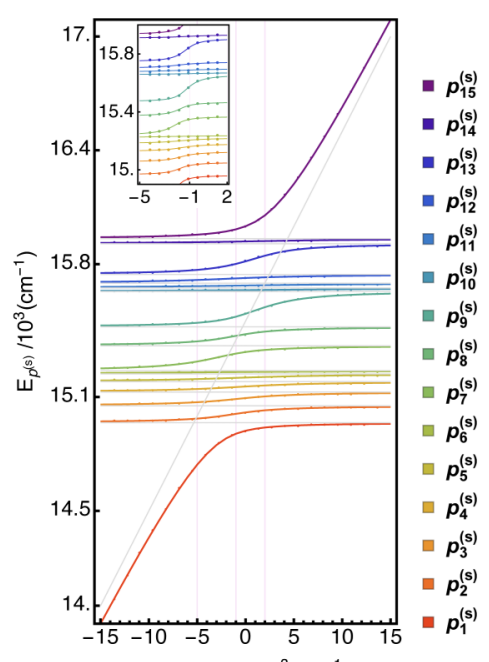

$\left(\omega_{c}-15500\right) / 10^{3}\left(\mathrm{~cm}^{-1}\right)$
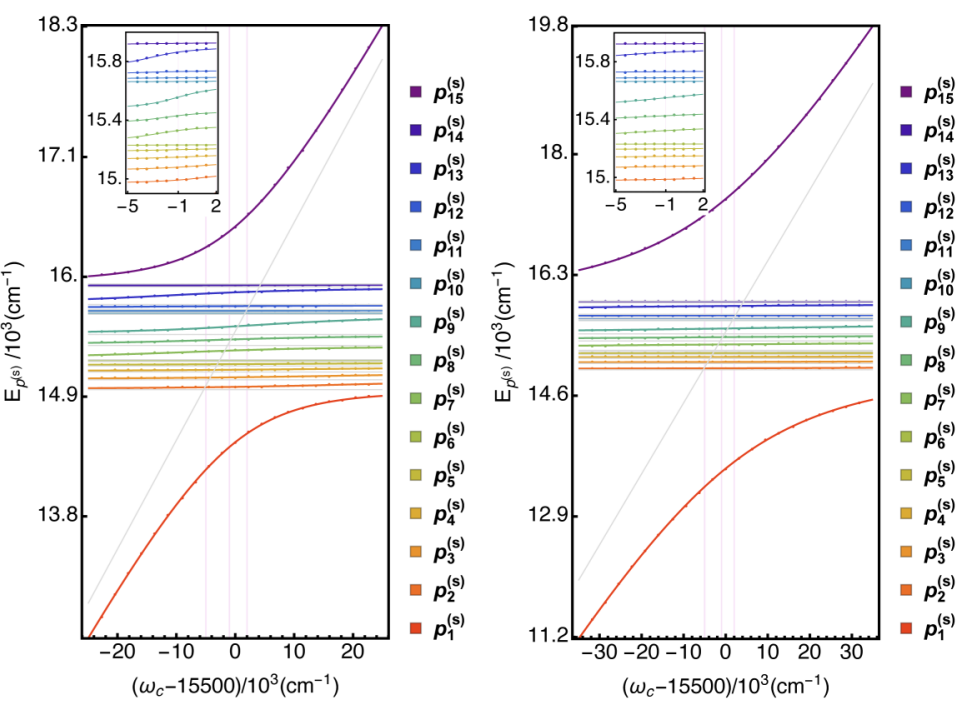

FIG. 2. The progression of bare polariton energy levels with respect to the cavity frequencies is presented, with the parametric variation of the cavity coupling strengths $\left(g_{c}^{l}-g_{c}^{\text {III }}\right.$, from the right to left) in between panels. The insets, in each panel, display the spectrally congested energy ranges [i.e., $\left.\Delta E_{p(s)}\left(\mathrm{cm}{ }^{-1}\right) \in\{14.9,16.0\} \times 10^{3}\right]$ The vertical lines represent the cavity frequencies $\omega_{c}^{\mathrm{I}}-\omega_{c}^{\text {III }}$ used in the simulation while the light gray lines denote the reference uncoupled cavity and exciton energy levels. 
(A)

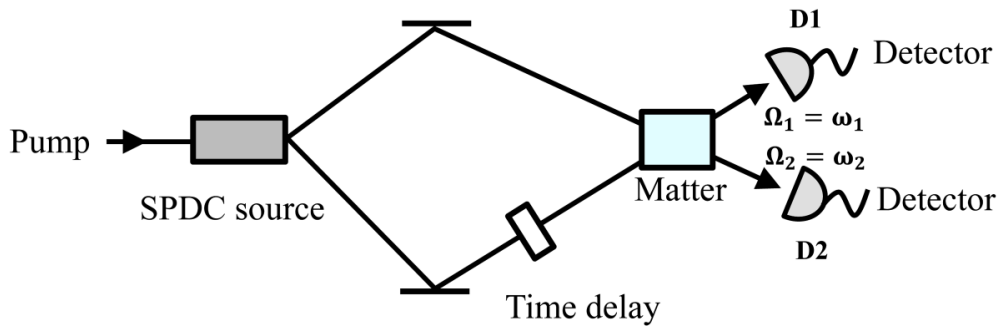

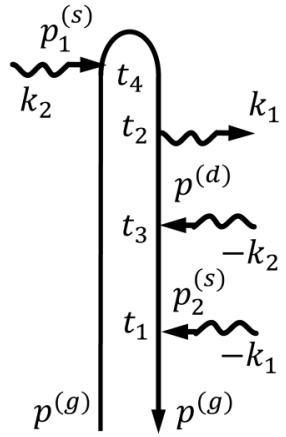

(B1)

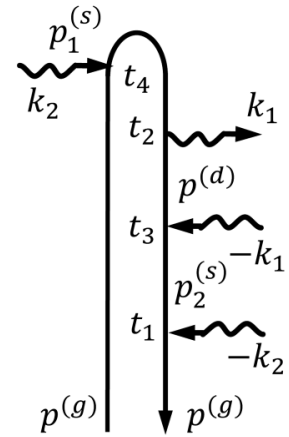

(B2)

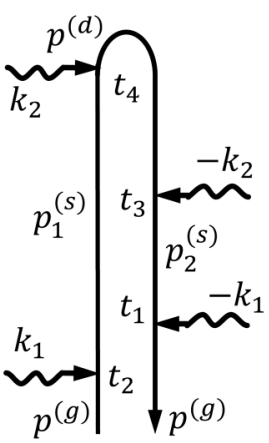

(B3)

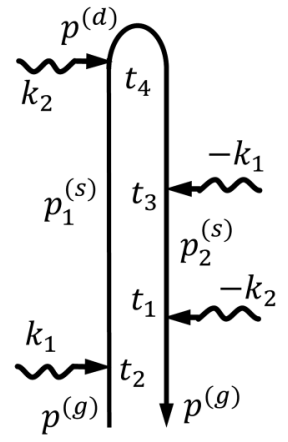

(B4)
FIG. 3. (a) The schematic setup of the proposed signal measurement technique is presented where the detector $D_{1}\left(D_{2}\right)$ monitors frequency at $\omega_{1}\left(\omega_{2}\right)$. In this work, the time delay has been set to zero. (b1)-(b4) The superoperator Schwinger-Keldysh diagrams for the corresponding polariton space pathways which describe the processes contributing to the signal are displayed. The rules for the diagrammatics have been described in the text. interchanged. The monitored signal, $S\left(\Omega_{2}, \Omega_{1}\right)$, is defined as the rate of change of the photon number operator in the transmitted mode at the detector $D_{2}\left(E_{D_{2}}\right)$, where the parametric frequencies $\Omega_{1}\left(\Omega_{2}\right)$ corresponds to the monitoring at detectors $D_{1}\left(D_{2}\right)$, as shown in the scheme in Fig. $3 .^{50}$ Following a straightforward manipulation, we have, $S\left(\Omega_{2}, \Omega_{1}\right)=-\operatorname{Im} 4 N \int_{-\infty}^{\infty} d t_{4}\left\langle E_{D_{2}}^{\dagger}\left(t_{4}\right) P^{(3)}\left(t_{4}\right)\right\rangle$, where the $P^{(3)}(t)$ is the time-dependent polarization operator expanded to the third order in the polariton-biphoton source field interaction. The averaging is carried out over the joint initial state configuration of the polariton and biphoton state. The parameter $N$, the number density of the emitters (cavity confined quantum aggregates), is taken as a scaling parameter in this work. The signal is evaluated algebraically by carrying out the dual-field perturbation expansion of the polarization operator ${ }^{51,52}$ which yields

$$
\begin{aligned}
S\left(\Omega_{2}, \Omega_{1}\right)= & \operatorname{Im} 4 N(i)^{3} \int_{-\infty}^{\infty} d t_{4} \int_{-\infty}^{\infty} d t_{3} \int_{-\infty}^{\infty} d t_{2} \int_{-\infty}^{\infty} d t_{1} \\
& \times \theta\left(t_{42}\right) \theta\left(t_{23}\right) \theta\left(t_{31}\right)\left\langle\tilde{X}\left(t_{1}\right) \tilde{X}\left(t_{3}\right) \tilde{X}^{\dagger}\left(t_{2}\right) \tilde{X}^{\dagger}\left(t_{4}\right)\right\rangle\left\langle E^{\dagger}\left(t_{1}\right) E^{\dagger}\left(t_{3}\right)\right. \\
& \left.\times E\left(t_{2}\right) E\left(t_{4}\right)\right\rangle+\theta\left(t_{42}\right) \theta\left(t_{21}\right) \theta\left(t_{13}\right)\left\langle\tilde{X}\left(t_{3}\right) \tilde{X}\left(t_{1}\right) \tilde{X}^{\dagger}\left(t_{2}\right) \tilde{X}^{\dagger}\left(t_{4}\right)\right\rangle \\
& \times\left\langle E^{\dagger}\left(t_{3}\right) E^{\dagger}\left(t_{1}\right) E\left(t_{2}\right) E\left(t_{4}\right)\right\rangle+\theta\left(t_{42}\right) \theta\left(t_{43}\right) \theta\left(t_{31}\right)\left\langle\tilde{X}\left(t_{1}\right) \tilde{X}\left(t_{3}\right)\right. \\
& \left.\times \tilde{X}^{\dagger}\left(t_{4}\right) \tilde{X}^{\dagger}\left(t_{2}\right)\right\rangle\left\langle E^{\dagger}\left(t_{1}\right) E^{\dagger}\left(t_{3}\right) E\left(t_{4}\right) E\left(t_{2}\right)\right\rangle+\theta\left(t_{42}\right) \theta\left(t_{41}\right) \theta\left(t_{13}\right) \\
& \times\left\langle\tilde{X}\left(t_{3}\right) \tilde{X}\left(t_{1}\right) \tilde{X}^{\dagger}\left(t_{4}\right) \tilde{X}^{\dagger}\left(t_{2}\right)\right\rangle\left\langle E^{\dagger}\left(t_{3}\right) E^{\dagger}\left(t_{1}\right) E\left(t_{4}\right) E\left(t_{2}\right)\right\rangle .
\end{aligned}
$$

In the above, the Heaviside functions, $\theta\left(t_{i j}\right)=\theta\left(t_{i}-t_{j}\right)$, determine the ordering among the successive polariton-biphoton interactions in each convolutional term, alternatively termed as pathways. The signal evaluation can be aided by the modular, dual-field diagrammatic notation, as shown in Fig. 3. Here, the evolution of the dual-Hilbert space fields is represented on the contour that keeps track of the contour-time variables. The polariton-biphoton source field interactions are represented via the wiggly external lines going into (away from) the loop denoting polariton excitation (deexcitation) processes. The interaction with the signal mode occurs at the end of the left-branch of the contour as a matter of convention. Field interactions within a branch of the contour are time-ordered. The intervals between the two interactions occurring the left(right) branch involve a retarded(advanced) polariton propagator $G_{p^{(n)} p^{(g)}}(\omega)\left(G_{p^{(n)} p^{(g)}}^{\dagger}(\omega)\right)$ The frequency arguments of the polariton Green's function cumulatively account for all the previous interactions. The different diagrams are generated by considering permutations of all the inter-branch interactions other than the one associated with the signal generation. In the signal evaluation above, we only focused on the set of pathways where the successive polariton-biphoton interactions of the type, $\left\langle E^{\dagger}\left(\tau_{4}\right) E^{\dagger}\left(\tau_{3}\right) E\left(\tau_{2}\right) E\left(\tau_{1}\right)\right\rangle$, were included. The neglected Raman interaction pathways (i.e., interactions of the type, $\left.\left\langle E^{\dagger}\left(\tau_{4}\right) E\left(\tau_{3}\right) E^{\dagger}\left(\tau_{2}\right) E\left(\tau_{1}\right)\right\rangle\right)$ offer additional insights into the dephasing mechanism induced by the polariton-polariton interactions. The latter may be observed by varying the corresponding photon-photon hole entanglement in the biphoton source field. ${ }^{53,54}$

\section{A. Entangled biphoton source properties}

The entangled biphoton sources can be generated by inducing a higher-order nonlinear interaction, namely, the spontaneous parametric downconversion (SPDC) process within the birefringent material $^{55,56}$ via a suitable classical pump pulse. The SPDC results 
in the emission of entangled biphotons, which are spectrally correlated in a non-classical manner within a specific temporal window. These spectral and temporal correlation properties may be examined and characterized by employing the photon coincidence counting measurements. ${ }^{57}$ To this end, we introduce the biphoton field amplitude function as $\left|\psi^{(2)}\right\rangle=\left(\chi^{(2)} / \sqrt{A}\right) E_{p} \sum_{k_{1}, k_{2}}$ sinc $\left(\Delta \omega t_{d} / 2\right) \exp \left(-i \Delta \omega t_{d} / 2\right) \operatorname{sinc}\left(\Delta k \times L_{z} / 2\right) \exp \left(-i \Delta k L_{z} / 2\right)\left|k_{1}, k_{2}\right\rangle$, where the biphoton field state $\left|k_{1}, k_{2}\right\rangle$ is defined over the nonseparable states (indexed by their modes $k_{i}$ ) satisfying the quasi-phase-matching condition $\Delta k=k_{p}-k_{1}-k_{2}$. The amplitude function encodes crucial information about the entangled biphoton generation process. It is parameterized by the nonlinear susceptibility of the material $\left(\chi^{(2)}\right)$, length of the SPDC crystal along the propagation direction $\left(L_{z}\right)$, the entanglement volume $(A)$, the mean interaction time within the crystal $\left(t_{d}\right)$, and the pump pulse envelope function $\left(E_{p}\right)$. The latter makes an allowance for the range of the frequencies $\left(\Delta \omega=\omega_{p}-\omega_{1}-\omega_{2}\right)$ over which the biphoton spectrum can be spread. Together, they determine the quantitative nature of the entanglement properties in the joint spectral-temporal domain. The introduction of the amplitude function further allows for the factorization of the multi-point biphoton field correlation functions using the following properties, $\langle$ vac $| E\left(t_{1}\right)$ $E\left(t_{2}\right)\left|\psi^{(2)}\right\rangle=F\left(t_{1}, t_{2}\right)+F\left(t_{2}, t_{1}\right) \quad$ and $\quad\left\langle\psi^{(2)}\left|E^{\dagger}\left(t_{1}\right) E^{\dagger}\left(t_{2}\right)\right| v a c\right\rangle=$ $F^{*}\left(t_{1}, t_{2}\right)+F^{*}\left(t_{2}, t_{1}\right)$. In the above, the auxiliary function $F\left(t_{1}, t_{2}\right)$ is defined as $F\left(t_{i}, t_{j}\right)=\sum_{i j} \frac{2 \pi \chi^{(2)} E_{p}}{V} \sqrt{\frac{\omega_{1} \omega_{2}}{A T_{b}}} \operatorname{rect} \frac{\left(t_{i}-t_{j}\right)}{T_{b}} \times e^{-i\left(\omega_{i} t_{i}\right)} e^{-i\left(\omega_{j} t_{j}\right)}$, where the $\omega_{i}\left(\omega_{j}\right)$ are the biphoton frequencies, $T_{b}=\left(1 / v_{1}-1 / v_{2}\right) L_{z}$ is the entanglement time where the material-dependent group velocities of the frequency components of the biphoton field within the crystal are denoted as $v_{1}\left(v_{2}\right)$, and the shifted rectangle function is defined as $\operatorname{rect}(x)=1$, for $0 \leq x \leq 1$ and $\operatorname{rect}(x)=0$, outside the range. The factorization properties of the field correlation functions determine the manner in which the bare polariton Green's functions are correlated with the biphoton field. The components of the polariton Green's functions represent the spectral amplitudes associated with the polariton modes. Therefore, the entanglement time $T_{b}$ can be used to govern the redistribution of the spectral amplitudes arising from different propagation intervals and to provide a way for exerting control over the correlation between the different polariton modes. The ability to parametrically manipulate the biphoton field correlations to achieve control over the desired components of the polariton Green's functions remain the central aim of the correlated spectroscopies such as the one described in this study.

In order to find an expression for the signal that explicitly demonstrates the differential manner of interaction between the amplitudes of the entangled biphoton fields and the polariton modes, the signal in Eq. (3) can be represented in the following form:

$$
\begin{aligned}
S\left(\Omega_{2}, \Omega_{1}\right)= & \operatorname{Im} 4 N(i)^{3} \int_{-\infty}^{\infty} d t_{4} \int_{-\infty}^{\infty} d t_{3} \int_{-\infty}^{\infty} d t_{2} \int_{-\infty}^{\infty} d t_{1} \\
& \times \theta\left(t_{42}\right) \theta\left(t_{23}\right) \theta\left(t_{31}\right)\left\langle\tilde{X}\left(t_{1}\right) \tilde{X}\left(t_{3}\right) \tilde{X}^{\dagger}\left(t_{2}\right) \tilde{X}^{\dagger}\left(t_{4}\right)\right\rangle F^{*}\left(t_{3}, t_{1}\right) F\left(t_{4}, t_{2}\right)+\theta\left(t_{42}\right) \theta\left(t_{21}\right) \theta\left(t_{13}\right)\left\langle\tilde{X}\left(t_{3}\right) \tilde{X}\left(t_{1}\right) \tilde{X}^{\dagger}\left(t_{2}\right) \tilde{X}^{\dagger}\left(t_{4}\right)\right\rangle \\
& \times F^{*}\left(t_{1}, t_{3}\right) F\left(t_{4}, t_{2}\right)+\theta\left(t_{42}\right) \theta\left(t_{43}\right) \theta\left(t_{31}\right)\left\langle\tilde{X}\left(t_{1}\right) \tilde{X}\left(t_{3}\right) \tilde{X}^{\dagger}\left(t_{4}\right) \tilde{X}^{\dagger}\left(t_{2}\right)\right\rangle F^{*}\left(t_{3}, t_{1}\right) F\left(t_{2}, t_{4}\right)+\theta\left(t_{42}\right) \theta\left(t_{41}\right) \\
& \times \theta\left(t_{13}\right)\left\langle\tilde{X}\left(t_{3}\right) \tilde{X}\left(t_{1}\right) \tilde{X}^{\dagger}\left(t_{4}\right) \tilde{X}^{\dagger}\left(t_{2}\right)\right\rangle F^{*}\left(t_{1}, t_{3}\right) F\left(t_{2}, t_{4}\right) .
\end{aligned}
$$

In this expression, the corresponding multi-time polariton space correlation functions are weighted by the biphoton correlation functions with different time arguments. Signatures of the interference between these pathways are encoded in the final signal. The magnitude of interference contributions are related to the enhancement or suppression of the individual polariton Green's function components, which will be explored in detail in Sec. III B.

\section{B. Multidimensional signal}

The role of the entangled biphoton field can be made more transparent by evaluating the multidimensional convolution integrals appearing in Eq. (4) using the form of biphoton correlations function introduced above. Toward that aim, we introduce the biphoton field modulated polariton Green's function, $\left.i \tilde{G}_{p^{(n)} p^{(g)}}\left(\omega, T_{b}\right)=\int_{\infty}^{\infty} d s e^{i \omega s} G_{p^{(n)} p^{(g)}}(s) \operatorname{rect}\left(s / T_{b}\right)=\left[\exp \left(i z_{p^{(n)} p^{(g)}}\right) T_{b}\right)-1\right] /$ $z_{p^{(n)} p^{(g)}}$, where the $G_{p^{(n)} p^{(g)}}(s)=\theta(s) \exp \left(-i H_{p^{(n)} p^{(g)}} s-\gamma_{\left.p^{(n)} p^{(g)} s\right)}\right.$ is the time domain bare polariton Green's function related to its frequency domain counterpart via the relation, $i G(\omega)=\int_{-\infty}^{\infty} d s \exp (i \omega s) G(s)$. $z_{p^{(n)} p^{(g)}}=\left(\omega-\omega_{p^{(n)} p^{(g)}}+i \gamma_{p^{(n)} p^{(g)}}\right)$ is the corresponding term that captures the dephasing broadened polariton resonances corresponding to the frequency domain polariton Green's function. The complicated yet discernible appearance of the resonances weighted by the entanglement time in the expression of $\tilde{G}_{p^{(n)} p^{(g)}}\left(\omega, T_{b}\right)$ determines the functional dependence of the biphoton field modulated polariton Green's functions and highlights the probe field's role in allowing specific resonances related to Green's function to be amplified or attenuated. The state specific dephasing parameters, given by $\gamma_{p^{(n)} p^{(g)}}=\Gamma_{p^{(n)} p^{(g)}}+\gamma_{p^{(n)} p^{(g)}}^{*}$, which systematically accounts for the linebroadening effects arising from intra-manifold polariton transport effects via the terms $\Gamma_{p^{(n)} p^{(g)}}$. The latter is related to the polariton transport matrix elements, which can be obtained from the dissipation kernel of the kinetic equation that governs the dynamics of the polariton states as described in the Appendix A. Introduction of biphoton field modulated polariton Green's functions into the Eq. (4) lead to the identification of the parametric frequencies (i.e., $\left.\Omega_{2}=\omega_{2}, \Omega_{1}=\omega_{1}\right)$ as the central frequencies associated with the entangled biphotons. The parametric frequencies $\Omega_{2}=\omega_{2}, \Omega_{1}=\omega_{1}$ serve as the scanning parameter for the signal. Finally, the compact sum over states expression of the signal is given as 


$$
\begin{aligned}
S\left(\Omega_{2}=\omega_{2}, \Omega_{1}=\omega_{1}\right)= & \sum_{p^{(g)}, p_{1}^{(s)} p_{2}^{(s)} p^{(d)}} C \times \omega_{1} \omega_{2} \operatorname{Im} d_{p_{1}^{(s)} p^{(g)}}^{*} d_{p_{2}^{(s)} p^{(g)}} d_{p_{2}^{(s)} p^{(d)}} d_{p_{1}^{(s)} p^{(d)}}^{*}\left(\tilde{G}_{p_{2}^{(s)} p^{(g)}}^{\dagger}\left(\omega_{1}, T_{b}\right) G_{p^{(d)} p^{(g)}}^{\dagger}\left(\omega_{1}+\omega_{2}\right) \tilde{G}_{p_{1}^{(s)} p^{(g)}}^{\dagger}\left(\omega_{2}, T_{b}\right)\right. \\
& +\tilde{G}_{p_{2}^{(s)} p^{(g)}}^{\dagger}\left(\omega_{2}, T_{b}\right) G_{p^{(d)} p^{(g)}}^{\dagger}\left(\omega_{1}+\omega_{2}\right) \tilde{G}_{p_{1}^{(s)} p^{(g)}}^{\dagger}\left(\omega_{2}, T_{b}\right)+\tilde{G}_{p_{2}^{(s)} p^{(g)}}^{\dagger}\left(\omega_{1}, T_{b}\right) G_{p^{(d)} p^{(g)}}^{\dagger}\left(\omega_{1}+\omega_{2}\right) \tilde{G}_{p_{1}^{(s)} p^{(g)}}\left(\omega_{1}, T_{b}\right) \\
& \left.+\tilde{G}_{p_{2}^{(s)} p^{(g)}}^{\dagger}\left(\omega_{2}, T_{b}\right) G_{p^{(d)} p^{(g)}}^{\dagger}\left(\omega_{1}+\omega_{2}\right) \tilde{G}_{p_{1}^{(s)} p^{(g)}}\left(\omega_{1}, T_{b}\right)\right) .
\end{aligned}
$$

In the above expressions, the collective coefficient $C=$ $16 \pi^{2} N\left|\chi^{(2)}\right|^{2}\left|E_{p}\right|^{2} / A T_{b} V^{2}$ encodes the properties of the classical laser pump field (i.e., the pump envelope function, $E_{p}$ ), the birefringent material responsible for the SPDC process (i.e., the material susceptibility, $\left.\chi^{(2)}\right)$, the entanglement time $\left(T_{b}\right)$, certain polariton ensemble characteristics (i.e., the number density of the aggregates, $N$ ), and the mode volume of the cavity, $V$. In the numerical simulation, while conducting the parametric comparison of the signals, the collective coefficient has been taken as a scaling factor.

The results presented in Figs. 4-6, as mentioned in their respective descriptions, show three sets of simulation outcomes, comprised of nine figures each. The sets themselves differ from each other by the values of the cavity mode frequencies. The values of the cavity mode frequencies, $\omega_{c}^{\mathrm{I}}-\omega_{c}^{\mathrm{III}}$ are chosen to be in resonance with the lower energy, the central region, and the higher energy region of the spectral bands of the bare exciton. ${ }^{32}$
Moreover, within a set, each column is characterized by different values for the entanglement times given as $T_{b}^{\mathrm{I}}=100 \mathrm{fs}, T_{b}^{\mathrm{II}}=200 \mathrm{fs}$, $T_{b}^{\mathrm{III}}=500 \mathrm{fs}$ while the rows account for the variations of the coupling strengths. In other words, in the simulation, each representative parameter combinations $\left\{\omega_{c}^{\mathrm{I}}, g_{c}^{\mathrm{I}}\right\}$ to $\left\{\omega_{c}^{\mathrm{III}}, g_{c}^{\mathrm{III}}\right\}$ is studied for three further variations of the biphoton entanglement times and has been presented along each column, i.e., making an assignment $\left\{\omega_{c}^{\mathrm{I}}, g_{c}^{\mathrm{I}}, T_{b}^{\mathrm{I}}\right\}$ for a particular set.

In the figures, the spectral distortion of the sidebands as one scans the coupling strengths from the lower to higher values, i.e., $g_{c}^{\mathrm{I}}-g_{c}^{\mathrm{III}}$, the existence of which can be inferred from the Fig. 2, is seen to be more pronounced while moving along the rows, for each value of the biphoton entanglement times. The scan over the coupling strengths makes the spectral sidebands appear at increasingly distant frequencies, as noted in Fig. 2, which participate in the dynamics by introducing novel multiple-scale dephasing pathways. The gradual onset of these spectral complexities is
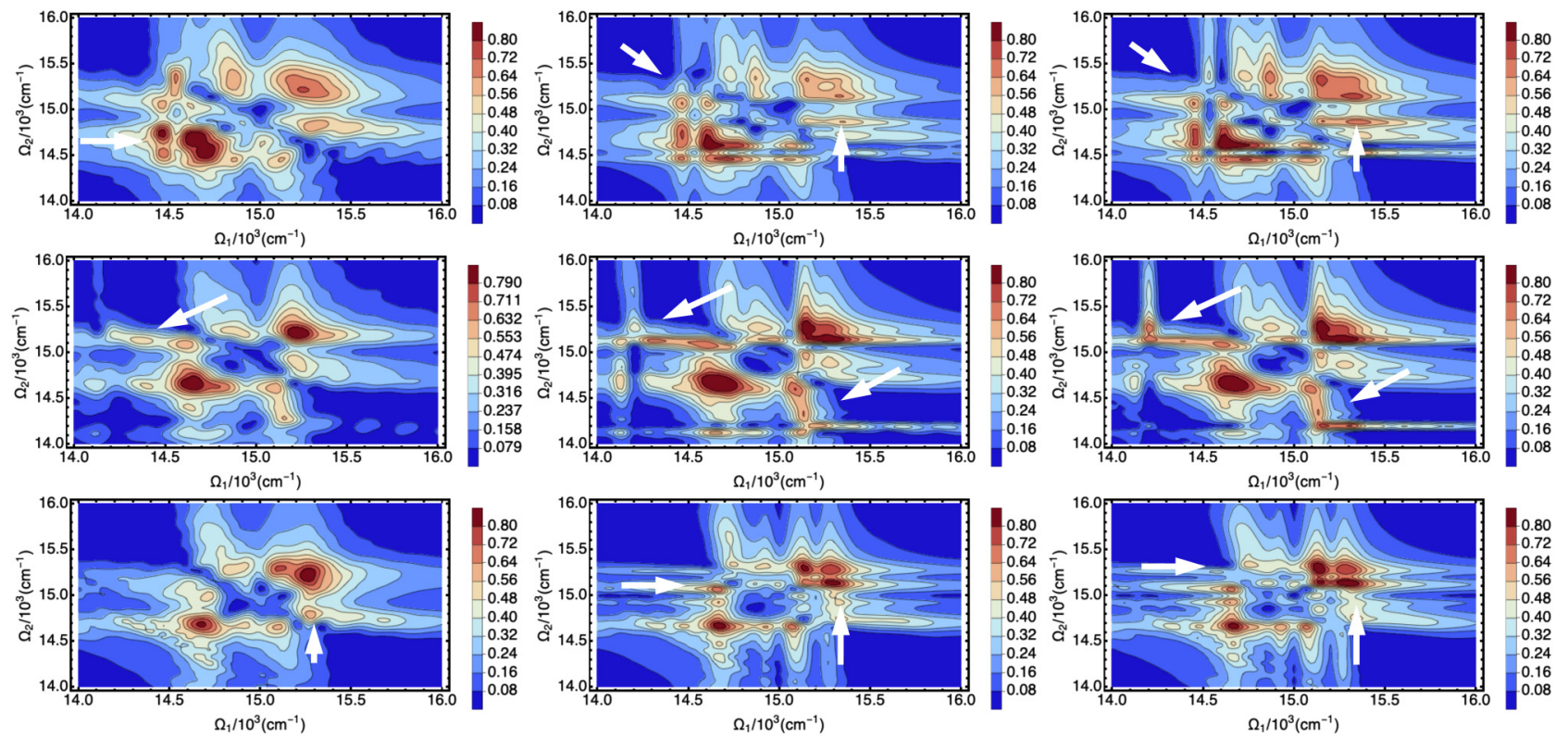

FIG. 4. The entangled biphoton assisted multidimensional spectra $S\left(\Omega_{2}=\omega_{2}, \Omega_{1}=\omega_{1}\right)$ have been displayed for the parametric cases with the assignments $\left\{\omega_{c}^{\perp}, g_{c}^{\mid}\right\},\left\{\omega_{c}^{\mid}, g_{c}^{\mid l}\right\}$, and $\left\{\omega_{c}^{!}, g_{c}^{|l|}\right\}$, along the rows, for the three variations of biphoton entanglement times, along the columns. The characteristics of some spectral regions whose qualitative changes have been discussed in the text are indicated by the arrows. 

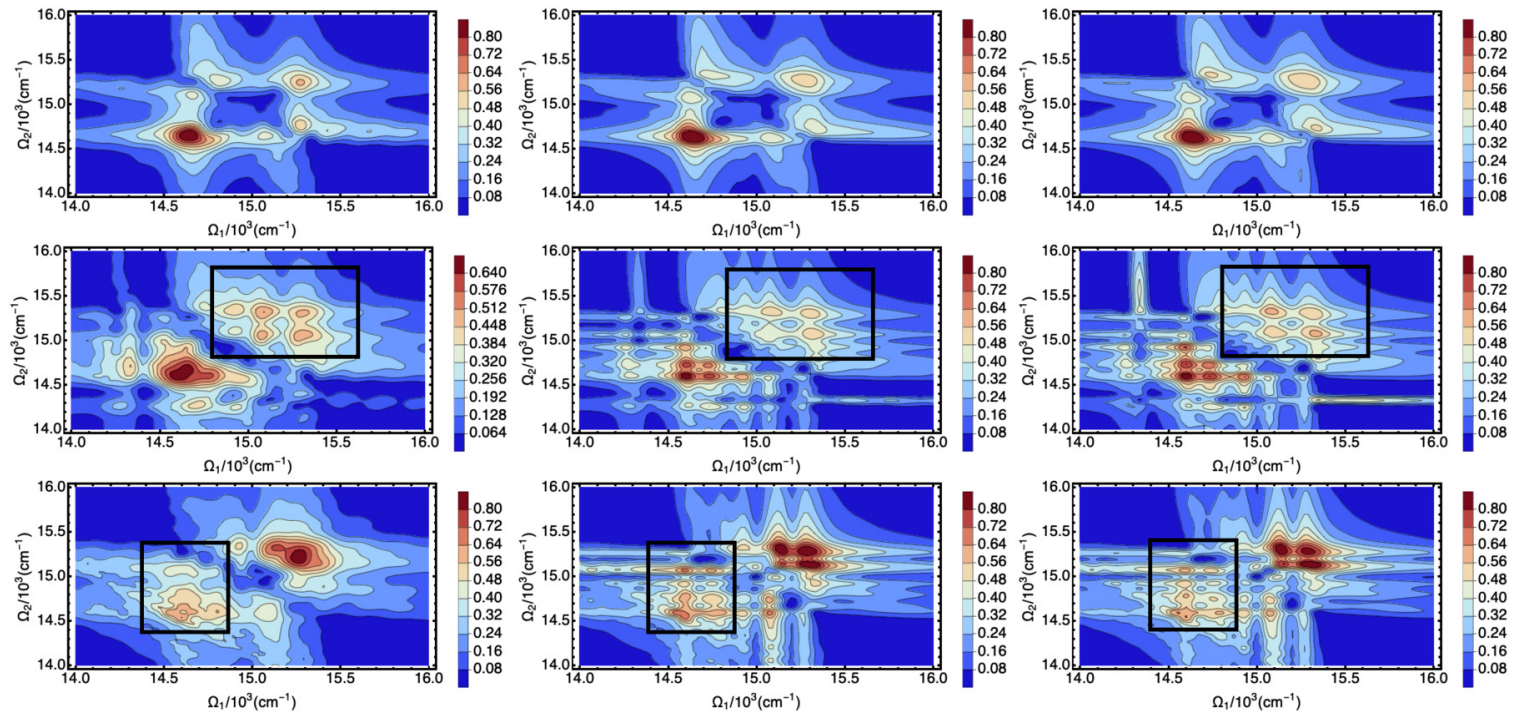

FIG. 5. The entangled biphoton assisted multidimensional spectra $S\left(\Omega_{2}=\omega_{2}, \Omega_{1}=\omega_{1}\right)$ have been shown for the parametric cases with the assignments $\left\{\omega_{c}^{\| \prime}, g_{c}^{\prime}\right\},\left\{\omega_{c}^{\|}, g_{c}^{\| \prime}\right\}$, and $\left\{\omega_{c}^{\|}, g_{c}^{|I|}\right\}$, along the rows, for the three variations of biphoton entanglement times, along the columns. The spectroscopic features relevant for the discussions displaying spectral beating and emergent spectral complexity have been highlighted.

seen by the appearance of the multiple off-diagonal spectral features while moving along rows in all the figures (e.g., $\left\{\omega_{c}^{\mathrm{III}}, g_{c}^{\mathrm{I}}, T_{b}^{\mathrm{I}}\right\}$ to $\left.\left\{\omega_{c}^{\mathrm{III}}, g_{c}^{\mathrm{III}}, T_{b}^{\mathrm{I}}\right\}\right)$ in Fig. 6 . Further, it can be seen that the offdiagonal peaks undergo qualitative changes as the biphoton entanglement time varies, e.g., $\left\{\omega_{c}^{\mathrm{I}}, g_{c}^{\mathrm{I}-\mathrm{III}}, T_{b}^{\mathrm{I}}\right\}$ to $\left\{\omega_{c}^{\mathrm{I}}, g_{c}^{\mathrm{I}-\mathrm{III}}, T_{b}^{\mathrm{III}}\right\}$ in Fig. 4 and quantitative changes, e.g., $\left\{\omega_{c}^{\mathrm{II}}, g_{c}^{\mathrm{II}-\mathrm{III}}, T_{b}^{\mathrm{I}}\right\}$ to $\left\{\omega_{c}^{\mathrm{II}}, g_{c}^{\mathrm{II}-\mathrm{III}}, T_{b}^{\mathrm{III}}\right\}$ in Fig. 5, and $\left\{\omega_{c}^{\mathrm{III}}, g_{c}^{\mathrm{I}-\mathrm{III}}, T_{b}^{\mathrm{I}}\right\}$ to $\left\{\omega_{c}^{\mathrm{III}}, g_{c}^{\mathrm{I}-\mathrm{III}}, T_{b}^{\mathrm{III}}\right\}$ in Fig. 6. They are indicative of the inter-manifold polariton
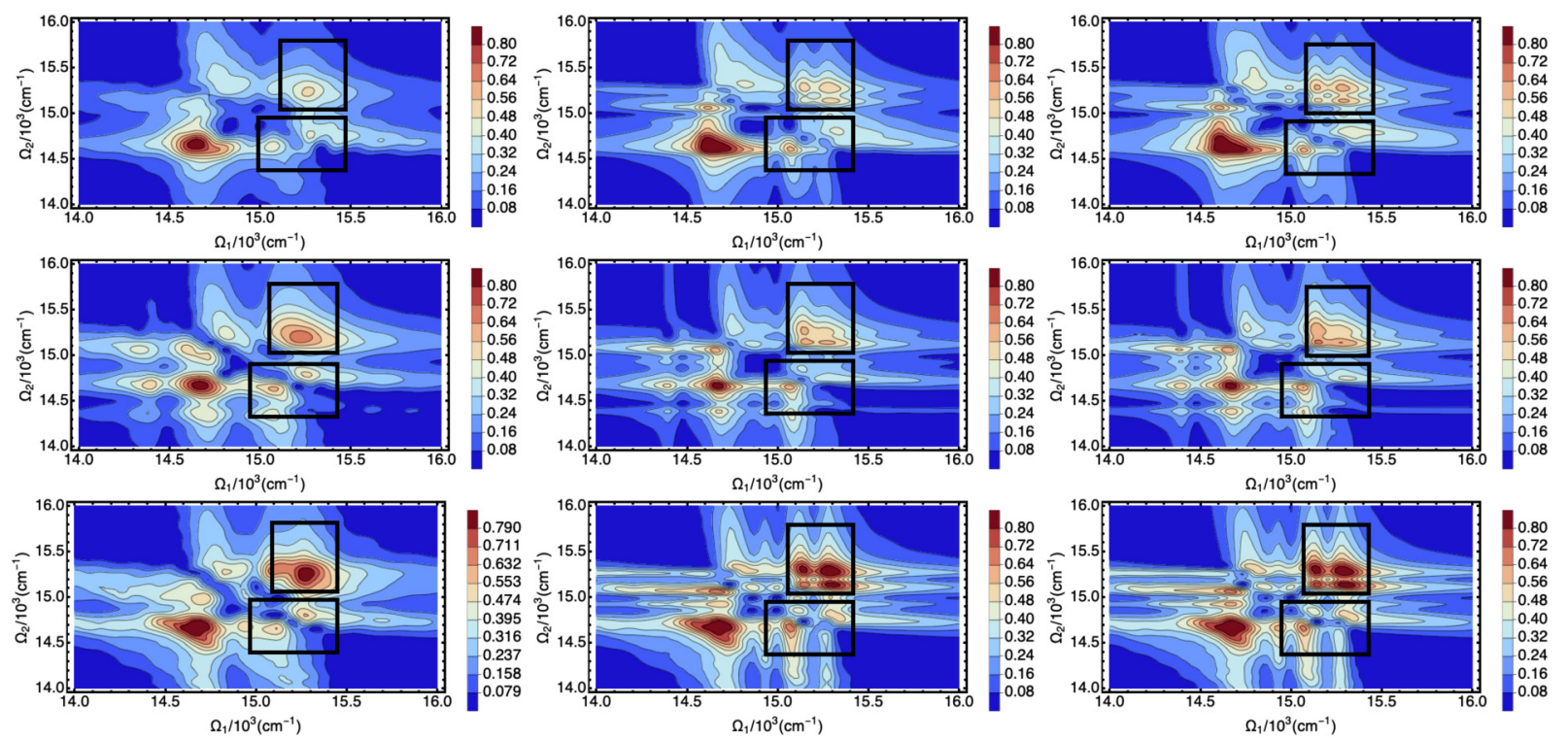

FIG. 6. The entangled biphoton assisted multidimensional spectra $S\left(\Omega_{2}=\omega_{2}, \Omega_{1}=\omega_{1}\right)$ have been presented for the parametric cases with the assignments $\left\{\omega_{c}^{\mathrm{III}}, g_{c}^{\prime}\right\},\left\{\omega_{c}^{\mathrm{III}}, g_{c}^{\mathrm{II}}\right\}$, and $\left\{\omega_{c}^{\mathrm{III}}, g_{c}^{\mathrm{III}}\right\}$, along the rows, for the three variations of biphoton entanglement times, along the columns. The spectroscopic features relevant for the discussions displaying spectral beating and emergent spectral complexity have been highlighted. Highlighted regions in the spectra offer corroborative evidence for the discussion regarding the quantitative changes in the off-diagonal features. 
coherence beats where the participating modes can be identified by the corresponding values in the diagonal. The corresponding frequencies indicated by the values in the axes can be recovered via suitable frequency gating of the detected signal. Specifically, these coherent features between correlated, spectrally distant modes may be discriminated by using biphoton fields with larger entanglement times for a chosen set of biphoton frequencies. The argument can be corroborated via observing the increased visibility of the certain off-diagonal features in the highlighted regions of the middle row in Fig. 5, and the upper row in Fig. 6 while moving along the columns. Crucially, in the former, the middle row, i.e., $\left\{\omega_{c}^{\mathrm{II}}, g_{c}^{\mathrm{II}}, T_{b}^{\mathrm{I}}\right\}$ to $\left\{\omega_{c}^{\mathrm{II}}, g_{c}^{\mathrm{II}}, T_{b}^{\mathrm{III}}\right\}$ demonstrates pronounced beating of the off-diagonal spectral features. Since the off-diagonal features in each quadrant of the frequency-correlation plots are indicative of polariton coherence signatures, it can be inferred that the longer entanglement times may be useful in the classification of the inter-manifold coherent contributions among the spectral components, even though the shorter entanglement times provide a better time-frequency resolution, revealing novel pathways. It is also observed that at the higher coupling strengths, e.g., in the bottom-most rows of Figs. 4-6, the spectral content of the diagonal spectral-domain does not undergo significant changes.

\section{DISCUSSIONS AND OUTLOOK}

In this communication, we presented a theoretical proposal for the multidimensional pump-probe analog spectroscopy of the cavity confined quantum aggregates using systematic variations of the cavity and entanglement parameters of the external biphoton source field. Each of the presented cases would constitute an independent measurement scheme within a parameter regime where the quantum coherent effects are known to be manifested in the cavity-free quantum aggregates. The spectroscopic signature of the quantum coherent effects appear in the off-diagonal features of the multidimensional spectra. ${ }^{17,40}$ It has been shown that similar characteristics emerge in the analyzed polaritonic frequency-correlation plots, which specifically brings out the information about the coherent dynamical correlation among the polariton modes in the presence of phonon interaction. The multiple classical field interactions, although effective, explore a reduced parameter domain of the polariton kinetics in the joint time-frequency space and hence may not be capable of deciphering these features. Equation (5) and the numerical simulations, together, constitute the central result of this communication.

Toward that end, one may propose an improved representation of the signal that may resemble the traditional MDCS counterpart more closely by introducing $S\left(\Omega_{\tau}, \Omega_{T_{b}}\right)=$ $\int_{0}^{-\infty} d \tau \int_{0}^{-\infty} d T \exp \left(i \Omega_{T_{b}} T_{b}\right) \exp \left(i \Omega_{\tau} \tau\right) S\left(\tau, T_{b}\right)$. It will require the joint variation of the finite time delay $(\tau)$, e.g., in Fig. 3 , the entanglement time $\left(T_{b}\right)$, and a double Fourier transform with respect to the corresponding variables. Given the recent progress in the shaping of biphoton sources and advancement in the studies of multidimensional spectroscopies of condensed phase systems, the proposed scheme is within the realm of experimental implementation in the near future. ${ }^{58-60}$
It should also be noted that the system treated in this communication explores the aggregate-cavity interaction at a theoretical level where the interactions among the aggregate building blocks have been neglected. The spatially extended inter-aggregate interactions, in the appropriate parameter regime, can be a source of crucial local field corrections toward nonlinear responses from collective excitations. ${ }^{61}$ Such ensemble effects also alter the scaling of the signal strengths with the number of emitters.

An essential extension of the present work would involve a comparative study of the real-time evolution of the non-Markovian density operator. ${ }^{62-65}$ Such studies would enable a more transparent interpretation regarding the signatures of the dynamical interferences present in the observed spectra and provide a gateway to the verifiable quantum process tomographic schemes. ${ }^{66-69}$ Further developments in these directions are under way.

\section{ACKNOWLEDGMENTS}

This work was supported by the European Research Council (No. ERC-2015-AdG694097), the Cluster of Excellence CUI (Advanced Imaging of Matter) Deutsche Forschungsgemeinschaft, 925, and the Grupos Consolidados (No. IT1249-19).

\section{APPENDIX A: KINETIC EQUATION}

The dissipative polariton kinetics is described via a limiting form of the generalized master equation, which treats the polariton-phonon interactions within the perturbative second Born approximation and neglects the cavity dissipation. Since the present study is focused on the ultrafast character of the polariton kinetics and the exciton-cavity coupling strengths exceed the excitonphonon coupling within the parameter regime of interest, such approximations allow for a physically admissible solution via ensuring the positivity of the polariton density operator at all times. The exciton-phonon interactions manifest itself in the polariton basis as the intra-manifold relaxation and both intra-manifold and intermanifold dephasing. These phenomena can be captured via the field-free, operator kinetic equation for the polariton density operator $\rho$ presented below

$$
d \rho(t) / d t=-i L \rho(t)-\int_{-\infty}^{t} d t^{\prime} \Sigma^{(2)}\left(t-t^{\prime}\right) \rho\left(t^{\prime}\right) .
$$

In the above, the coherent evolution superoperator, $L \rho(s)=H \rho(s)$ $-\rho(s) H$ is defined via its commutator acting on the density operator, and the dissipation kernel superoperator, $\Sigma^{(2)}\left(t-t^{\prime}\right)$ is given by

$$
\Sigma^{(2)}\left(t-t^{\prime}\right) \rho\left(t^{\prime}\right)=-\left[X^{p}, D^{p}\left(t-t^{\prime}\right) \rho\left(t^{\prime}\right)-\rho\left(t^{\prime}\right) D^{p^{\dagger}}\left(t-t^{\prime}\right)\right] .
$$

The function, $D^{p}(t)=\int_{0}^{\infty} d t^{\prime} X^{p}\left(t-t^{\prime}\right) C\left(t-t^{\prime}\right)$, is defined as the convolution of the polariton-phonon coupling operator (in the Heisenberg representation), $\left(X^{p}(\tau)\right)$, and the finite temperature equilibrium correlation function of the phonons, $(C(\tau))$. The former, in the polariton basis, is given by 
$X_{p^{(n)} p^{\left(n^{\prime}\right)}}^{p}(\tau)=\sum_{n n^{\prime}} \exp \left(i \omega_{p^{(n)} p^{\left(n^{\prime}\right)}} \tau\right) X_{p^{(n)} p^{\left(n^{\prime}\right)}}$ while the latter is given by

$C(\tau)=\left(\lambda_{0} \gamma_{0} / 2\right) \cot \left(\beta \gamma_{0} / 2\right) \exp \left(-\gamma_{0} \tau\right)+\sum_{j=1}^{N_{b}} \frac{\lambda_{j}}{2 \zeta_{j}}$

$\times\left(\operatorname{coth}\left(i \beta \phi_{j}^{+} / 2\right) \exp \left(-\phi_{j}^{+} \tau\right)-\operatorname{coth}\left(i \beta \phi_{j}^{-} / 2\right) \exp \left(-\phi_{j}^{-} \tau\right)\right)$

$+\left(-i \lambda_{0} \gamma_{0} / 2\right) \exp \left(-\gamma_{0} \tau\right)+\frac{i \lambda_{j} v_{j}^{2}}{2 \zeta_{j}}\left(\exp \left(-\phi_{j}^{+} t\right)-\exp \left(-\phi_{j}^{-} t\right)\right)$

$-\sum_{n=1}^{\infty}\left(\left(4 \lambda_{j} \gamma_{j} v_{j}^{2} / \beta\right)\left(v_{n} /\left(v_{j}^{2}+v_{n}^{2}\right)^{2}-v_{n}^{2} \gamma_{j}^{2}\right)\right.$

$\left.+\left(2 \lambda_{0} \gamma_{0} / \beta\right)\left(v_{n} /\left(v_{n}^{2}-\gamma_{0}^{2}\right)\right)\right) \exp \left(-i v_{n} \tau\right)$,

where we have abbreviated, $\zeta_{j}=\sqrt{\left(v_{j}^{2}-\gamma_{j}^{2} / 4\right)}, \phi_{j}^{ \pm}=\left(\gamma_{j} / 2\right) \pm i \zeta_{j}$, and denoted $v_{n}=n(2 \pi / \beta)$ (where $\beta=1 / \kappa T$, with $\kappa$ and $T$ being the Boltzmann constant and the temperature) as Matsubara frequency terms, which are set at $n=20$. Subsequently, while evaluating the dissipative kernel, an assumption is made about the single time dependence of the density operator, i.e., $\rho\left(t-t^{\prime}\right) \approx \rho(t)$, the integration variable is changed from $t^{\prime}$ to $\tau=\left(t-t^{\prime}\right)$, and the upper limit is extended to infinity, leading to an effective Markovian limit. Due to the latter and the neglect of the effects of the driving on dissipation, the self-energy terms remain manifestly frequency-independent and the frequency domain Green's functions appear in a much simpler form. Finally, the relevant matrix elements of the dissipation kernel yield the polariton transport matrix defined as $\Sigma_{p^{(n)} p^{(n)} p^{\left(n^{\prime}\right)} p^{\left(n^{\prime}\right)}}^{(2)}$, whose limit $n=n^{\prime}$ yields the transport related line-broadening contributions to the polariton dephasing, as mentioned in the Sec. III B, via the relation, $\Gamma_{p^{(n)} p^{(g)}}=\frac{1}{2}\left(\Sigma_{p^{(n)} p^{(n)} p^{(n)} p^{(n)}}^{(2)}+\Sigma_{p^{(g)} p^{(g)} p^{(g)} p^{(g)}}^{(2)}\right)$. In the above, the mapping of the tetradic operators is made possible by their identical indices and the fact that ground polariton state does not participate in transport, i.e., $\Sigma_{p^{(g)} p^{(g)} p^{(g)} p^{(g)}}^{(2)}=0$.

\section{APPENDIX B: PHONON PROPERTIES}

Here, we present the numerical simulation parameters the 48 phonon modes, which have been used in the simulation (Tables I and II).

$\gamma_{j}\left(\mathrm{~cm}^{-1}\right)=30.0$ has been uniformly set for all the multimode Brownian oscillators. Analogously, the corresponding numerical values of the overdamped oscillator are taken as $\lambda_{0}\left(\mathrm{~cm}^{-1}\right)=37.0$ and $\gamma_{0}\left(\mathrm{~cm}^{-1}\right)=30.0$.

TABLE I. The list of the $v_{j}\left(\mathrm{~cm}^{-1}\right)$ parameters corresponding to the multi-mode Brownian oscillators.

\begin{tabular}{ccccccccc}
\hline \hline & 97 & 604 & 1143 & 1354 & 138 & 700 & 1181 & 1382 \\
& 213 & 722 & 1190 & 1439 & 260 & 742 & 1208 & 1487 \\
$v_{j}\left(\mathrm{~cm}^{-1}\right)$ & 298 & 752 & 1216 & 1524 & 342 & 795 & 1235 & 1537 \\
& 388 & 916 & 1252 & 1553 & 425 & 986 & 1260 & 1573 \\
& 518 & 995 & 1286 & 1580 & 546 & 1052 & 1304 & 1612 \\
& 573 & 1069 & 1322 & 1645 & 585 & 1110 & 1338 & 1673 \\
\hline \hline
\end{tabular}

TABLE II. The list of the $\lambda_{j}\left(\mathrm{~cm}^{-1}\right)$ parameters corresponding to the multi-mode Brownian oscillators.

\begin{tabular}{ccccccc}
\hline \hline & 2.32412 & 1.17176 & 46.7944 & 7.79904 & 3.97578 & 1.379 \\
& 20.7738 & 9.21794 & 6.39426 & 2.84468 & 7.9373 & 9.59813 \\
& 6.9394 & 29.2496 & 22.348 & 11.7176 & 7.95362 & 19.3866 \\
$\lambda_{j}\left(\mathrm{~cm}^{-1}\right)$ & 21.3894 & 9.69264 & 20.6397 & 3.85575 & 8.60795 & 33.5527 \\
& 9.64956 & 19.4467 & 7.96272 & 14.1168 & 6.3155 & 10.1657 \\
& 8.0136 & 7.14142 & 20.4196 & 22.6263 & 5.83844 & 7.1732 \\
1.46874 & 12.7608 & 7.51104 & 7.31848 & 4.86477 & 6.79884 \\
40.083 & 5.97135 & 1.77255 & 12.4542 & 5.27172 & 1.62281 \\
\hline \hline
\end{tabular}

\section{DATA AVAILABILITY}

The data that support the findings of this study are available from the corresponding author upon reasonable request.

\section{REFERENCES}

${ }^{1}$ R. Schnabel, Phys. Rep. 684, 1 (2017).

${ }^{2}$ L. Barsotti, J. Harms, and R. Schnabel, Rep. Prog. Phys. 82, 016905 (2019).

${ }^{3}$ M. J. Padgett and R. W. Boyd, Philos. Trans. R. Soc. A 375, 20160233 (2017).

${ }^{4}$ T. Aidukas, P. C. Konda, A. R. Harvey, M. J. Padgett, and P. A. Moreau, Sci. Rep. 9, 1 (2019).

${ }^{5}$ E. Toninelli, P.-A. Moreau, T. Gregory, A. Mihalyi, M. Edgar, N. Radwell, and M. Padgett, Optica 6, 347 (2019).

${ }^{6}$ S. Mukamel, M. Freyberger, W. Schleich, M. Bellini, A. Zavatta, G. Leuchs, C. Silberhorn, R. W. Boyd, L. L. Sánchez-Soto, A. Stefanov, M. Barbieri, A. Paterova, L. Krivitsky, S. Shwartz, K. Tamasaku, K. Dorfman, F. Schlawin, V. Sandoghdar, M. Raymer, A. Marcus, O. Varnavski, T. Goodson, Z.-Y. Zhou, B.-S. Shi, S. Asban, M. Scully, G. Agarwal, T. Peng, A. V. Sokolov, Z.-D. Zhang, M. S. Zubairy, I. A. Vartanyants, E. del Valle, and F. Laussy, J. Phys. B 53, $072002(2020)$.

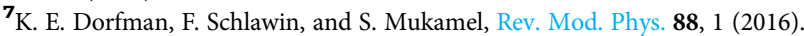

${ }^{8}$ F. Schlawin and A. Buchleitner, New J. Phys. 19, 013009 (2017).

${ }^{\mathbf{9}}$ F. Schlawin, K. E. Dorfman, B. P. Fingerhut, and S. Mukamel, Nat. Commun. 4, 1 (2013).

${ }^{10}$ L. Ye and S. Mukamel, Appl. Phys. Lett. 116, 174003 (2020).

${ }^{11}$ R. Fickler, R. Lapkiewicz, M. Huber, M. P. Lavery, M. J. Padgett, and A. Zeilinger, Nat. Commun. 5, 1 (2014).

${ }^{12}$ A. Pecoraro, F. Cardano, L. Marrucci, and A. Porzio, Phys. Rev. A 100, 1 (2019).

${ }^{13}$ A. M. Yao and M. J. Padgett, Adv. Opt. Photonics 3, 161 (2011).

${ }^{14}$ S. Mukamel, in Principles of Nonlinear Optical Spectroscopy, Optical and Imaging Sciences Series (Oxford University Press, 1995).

${ }^{15}$ S. Mukamel and D. Abramavicius, Chem. Rev. 104, 2073 (2004).

${ }^{16}$ D. Abramavicius, B. Palmieri, and D. V. Voronine, Spectroscopy 109, 2350 (2009).

${ }^{17}$ S. T. Cundiff and S. Mukamel, Phys. Today 66(7), 44 (2013).

${ }^{18}$ M. Cho, Chem. Rev. 108, 1331 (2008).

${ }^{19}$ F. Schlawin and S. Mukamel, J. Chem. Phys. 139, 244110 (2013).

${ }^{20}$ F. Schlawin, J. Phys. B 50, 203001 (2017).

${ }^{21}$ J. Svozilík, J. Peřina, and R. D. J. León-Montiel, J. Opt. Soc. Am. B 35, 460 (2018).

${ }^{22}$ R. D. J. León-Montiel, J. Svozilík, J. P. Torres, and A. B. U'Ren, Phys. Rev. Lett. 123, 023601 (2019).

${ }^{23}$ E. R. Bittner, H. Li, A. Piryatinski, A. R. Srimath Kandada, and C. Silva, J. Chem. Phys. 152, 071101 (2020). 
${ }^{24}$ F. Caruso, S. K. Saikin, E. Solano, S. F. Huelga, A. Aspuru-Guzik, and M. B. Plenio, Phys. Rev. B 85, 1 (2012).

${ }^{25}$ F. M. Marchetti and J. Keeling, Phys. Rev. Lett. 113, 216405 (2014).

${ }^{26}$ A. Shalabney, C. Genet, G. Pupillo, J. George, J. Hutchison, and T. W. Ebbesen, Nat. Commun. 6, 5981 (2015).

27. Flick, M. Ruggenthaler, H. Appel, and A. Rubio, Proc. Natl. Acad. Sci. U.S.A. 114, 3026 (2017)

${ }^{\mathbf{2 8}} \mathrm{G}$. Groenhof and J. J. Toppari, J. Phys. Chem. Lett. 9, 4848 (2018).

${ }^{29}$ C. Schäfer, M. Ruggenthaler, and A. Rubio, Phys. Rev. A 98, 043801 (2018).

${ }^{30}$ R. Sáez-Blázquez, J. Feist, E. Romero, A. I. Fernández-Domínguez, and F. J. García-Vidal, J. Phys. Chem. Lett. 10, 4252 (2019).

${ }^{31}$ Y. C. Chen, B. Song, A. J. Leggett, P. Ao, and X. Zhu, Phys. Rev. Lett. 122, 257402 (2019).

${ }^{32}$ Z. Zhang, P. Saurabh, K. E. Dorfman, A. Debnath, and S. Mukamel, J. Chem. Phys. 148, 074302 (2018).

${ }^{33}$ M. Schröter, S. D. Ivanov, J. Schulze, S. P. Polyutov, Y. Yan, T. Pullerits, and O. Kühn, Phys. Rep. 567, 1 (2015).

${ }^{34}$ F. Albert, K. Sivalertporn, J. Kasprzak, M. Strauß, C. Schneider, S. Höfling, M. Kamp, A. Forchel, S. Reitzenstein, E. A. Muljarov, and W. Langbein, Nat. Commun. 4, 1 (2013).

${ }^{35}$ J. Schachenmayer, C. Genes, E. Tignone, and G. Pupillo, Phys. Rev. Lett. 114, 196403 (2015).

${ }^{36}$ M. Kiffner, J. Coulthard, F. Schlawin, A. Ardavan, and D. Jaksch, New J. Phys. 21, 073066 (2019).

${ }^{37}$ X. Wei, X. Su, P. Cao, X. Liu, W. Chang, M. Li, X. Zhang, and Z. Liu, Nature 534, 69 (2016).

${ }^{38}$ G. S. Schlau-Cohen, A. Ishizaki, T. R. Calhoun, N. S. Ginsberg, M. Ballottari, R. Bassi, and G. R. Fleming, Nat. Chem. 4, 389 (2012).

${ }^{39}$ G. S. Schlau-Cohen, T. R. Calhoun, N. S. Ginsberg, M. Ballottari, R. Bassi, and G. R. Fleming, Proc. Natl. Acad. Sci. U.S.A. 107, 13276 (2010).

${ }^{40}$ J. Cao, R. J. Cogdell, D. F. Coker, H. G. Duan, J. Hauer, U. Kleinekathöfer, T. L. Jansen, T. Mančal, R. J. Dwayne Miller, J. P. Ogilvie, V. I. Prokhorenko, T. Renger, H. S. Tan, R. Tempelaar, M. Thorwart, E. Thyrhaug, S. Westenhoff, and D. Zigmantas, Sci. Adv. 6, 1 (2020).

${ }^{41} \mathrm{~V}$. Agranovich and B. Toshich, Sov. Phys. JETP 26, 104 (1968).

${ }^{42}$ V. Chernyak, N. Wang, and S. Mukamel, Phys. Rep. 263, 213 (1995).

${ }^{43}$ V. I. Novoderezhkin, M. A. Palacios, H. Van Amerongen, and R. Van Grondelle, J. Phys. Chem. B 108, 10363 (2004).

${ }^{44}$ V. Novoderezhkin, A. Marin, and R. Van Grondelle, Phys. Chem. Chem. Phys. 13, 17093 (2011).

${ }^{45}$ I. Carusotto and C. Ciuti, Rev. Mod. Phys. 85, 299 (2013).
${ }^{46}$ O. Di Stefano, A. Settineri, V. Macrì, L. Garziano, R. Stassi, S. Savasta, and F. Nori, Nat. Phys. 15, 803 (2019).

${ }^{47}$ A. Frisk Kockum, A. Miranowicz, S. De Liberato, S. Savasta, and F. Nori, Nat. Rev. Phys. 1, 19 (2019).

${ }^{48}$ C. Schäfer, M. Ruggenthaler, V. Rokaj, and A. Rubio, ACS Photonics 7, 975 (2020).

${ }^{49}$ A. Stokes and A. Nazir, Nat. Commun. 10, 499 (2019).

${ }^{50}$ O. Roslyak and S. Mukamel, Phys. Rev. A 79, 063409 (2009).

51 S. Mukamel, Phys. Rev. A 77, 023801 (2008).

${ }^{52}$ S. Mukamel, R. Oszwaldowski, and D. Abramavicius, Phys. Rev. B 75, 245305 (2007).

${ }^{53}$ J. D. Franson, Phys. Rev. Lett. 96, 1 (2006).

${ }^{54}$ T. B. Pittman and J. D. Franson, Phys. Rev. A 74, 1 (2006).

${ }^{55}$ B. E. Saleh, B. M. Jost, H. B. Fei, and M. C. Teich, Phys. Rev. Lett. 80, 3483 (1998).

${ }^{56}$ A. Christ, K. Laiho, A. Eckstein, K. N. Cassemiro, and C. Silberhorn, New J. Phys. 13, 033027 (2011).

${ }^{57}$ K. E. Dorfman and S. Mukamel, Phys. Rev. A 86, 023805 (2012).

${ }^{58}$ L. Qi, M. Manceau, A. Cavanna, F. Gumpert, L. Carbone, M. D. Vittorio, A. Bramati, E. Giacobino, L. Lachman, R. Filip, and M. Chekhova, New J. Phys. 20, 073013 (2018).

${ }^{\mathbf{5 9}}$ M. G. Raymer, A. H. Marcus, J. R. Widom, and D. L. Vitullo, J. Phys. Chem. B 117,15559 (2013).

${ }^{60}$ H. C. Chan, O. E. Gamel, G. R. Fleming, and K. B. Whaley, J. Phys. B 51, 054002 (2018).

${ }^{61}$ S. Schütz, J. Schachenmayer, D. Hagenmüller, G. K. Brennen, T. Volz, V. Sandoghdar, T. W. Ebbesen, C. Genes, and G. Pupillo, Phys. Rev. Lett. 124, $113602(2020)$.

${ }^{62}$ A. G. Dijkstra and Y. Tanimura, Phys. Rev. Lett. 104, 1 (2010).

${ }^{63}$ Y. Tanimura, J. Phys. Soc. Jpn. 75, 1 (2006).

${ }^{64}$ A. Debnath, C. Meier, B. Chatel, and T. Amand, Phys. Rev. B 86, 161304(R) (2012).

${ }^{65}$ A. Debnath, C. Meier, B. Chatel, and T. Amand, Phys. Rev. B 88, 201305 (2013).

${ }^{66}$ E. Harel, A. F. Fidler, and G. S. Engel, Proc. Natl. Acad. Sci. U.S.A. 107, 16444 (2010)

${ }^{67}$ R. Rey-De-Castro, R. Cabrera, D. I. Bondar, and H. Rabitz, New J. Phys. 15, 025032 (2013).

${ }^{68}$ J. Yuen-Zhou, J. J. Krich, M. Mohseni, and A. Aspuru-Guzik, Proc. Natl. Acad. Sci. U.S.A. 108, 17615 (2011).

${ }^{69}$ S. Hoyer and K. B. Whaley, J. Chem. Phys. 138, 164102 (2013). 